\title{
REGULARIZATION IN KELLER-SEGEL TYPE SYSTEMS AND THE DE GIORGI METHOD*
}

\author{
BENOîT PERTHAME ${ }^{\dagger}$ AND ALEXIS VASSEUR ${ }^{\ddagger}$
}

\begin{abstract}
Fokker-Planck systems modeling chemotaxis, haptotaxis, and angiogenesis are numerous and have been widely studied. Several results exist that concern the gain of $L^{p}$ integrability but methods for proving regularizing effects in $L^{\infty}$ are still very few.

Here, we consider a special example, related to the Keller-Segel system, which is both illuminating and singular by lack of diffusion on the second equation (the chemical concentration). We show the gain of $L^{\infty}$ integrability (strong hypercontractivity) when the initial data belongs to the scaleinvariant space.

Our proof is based on De Giorgi's technique for parabolic equations. We present this technique in a formalism which might be easier that the usual iteration method. It uses an additional continuous parameter and makes the relation to kinetic formulations for hyperbolic conservation laws.
\end{abstract}

Key words. De Giorgi method, entropy methods, regularizing effects, hypercontractivity, Keller-Segel system, haptotaxis.

AMS subject classifications. 35K55, 35B65, 92C17.

\section{Introduction}

The Keller-Segel 19 model is certainly the simplest and best known model of a nonlinear Fokker-Planck equation where the nonlinearity comes from the drift term. The fact that, despite the mass being globally conserved, singularities occur in finite time for large data while smooth solutions exist globally for small data [17, 25, 26, 20, 21, 22, 3] is both a generic property of conservative nonlinear PDEs and a symptom of the inherent mathematical difficulties of such problems.

Our first purpose here is to exemplify, in the case of a particularly singular coupling, the use of the De Giorgi method [13] for proving the gain of the $L^{\infty}$ property within the framework of such model. This is the reason why we prefer, in place of the Keller-Segel system, another Fokker-Planck equation more related to the modeling of haptotaxis and angiogenesis and which reads as

$$
\left\{\begin{array}{l}
\frac{\partial}{\partial t} n=\Delta n-\nabla \cdot[n \chi(c) \nabla c], \quad t>0, x \in \mathbb{R}^{d}, \\
\frac{\partial}{\partial t} c=-c n, \quad t>0, x \in \mathbb{R}^{d}, \quad c(0, x)=c^{0}(x) \geq 0, \quad x \in \mathbb{R}^{d} . \\
n(0, x)=n^{0}(x) \geq 0, \quad
\end{array}\right.
$$

Here $n(x, t)$ denotes the population density of cells moving according to biased random motion towards high values of a substance concentration denoted by $c(x, t)$ and which is consumed by the cells. We refer to [12, 15, 23, for more realistic models in this area and more details on the modeling aspects. The sensitivity $\chi(c)$ is a given smooth positive function on $\mathbb{R}_{+}$, generally chosen as a decreasing function since sensitivity is

* Received: February 10, 2011; accepted (in revised version): June 28, 2011. Communicated by Shi Jin.

†Université Pierre et Marie Curie Paris-6, UMR CNRS 7598 "Laboratoire Jacques-Louis Lion", BC187, 4 place Jussieu, F-75252 Paris cedex 5, and Institut Universitaire de France and INRIA Paris-Rocquencourt, team BANG (benoit.perthame@upmc.fr).

$\ddagger$ University of Oxford, Mathematical Institute, 24-29 St Giles’, Oxford, OX1 3LB England (Alexis.Vasseur@maths.ox.ac.uk). 
lower for higher concentrations of the chemical because of saturation effects; a related case with sensitivity $\chi=1 / c$ has a particularly interesting mathematical structure [18, 24].

Weak solutions to (1.1) are treated in [9, 10] and propagation of $L^{\infty}$ bounds in [11. For this model we prove the following theorem.

Theorem 1.1. Let $d \geq 2$. A classical solution to (1.1), with $c^{0} \in$ $L^{\infty}\left(\mathbb{R}^{d}\right)$ and $\left\|n^{0}\right\|_{L^{\frac{d}{2}\left(\mathbb{R}^{d}\right)}} \leq K\left(d,\left\|c^{0}\right\|_{\infty}\right)$ small enough, satisfies for some constant $C\left(d,\left\|n^{0}\right\|_{L^{\frac{d}{2}}\left(\mathbb{R}^{d}\right)},\left\|c^{0}\right\|_{L^{\infty}\left(\mathbb{R}^{d}\right)}\right)$

$$
\|n(t)\|_{L^{\infty}\left(\mathbb{R}^{d}\right)} \leq \frac{C(d)}{t} \quad \forall t>0 .
$$

This result expresses both the regularizing effect and time decay of the heat equation. Not only does it establish these properties for a more singular system than those used presently (parabolic or elliptic equations on $c$ ) but it also treats the critical space $L^{\frac{d}{2}}$ which frequently appears in the Keller-Segel type of models. Indeed $L^{\frac{d}{2}}$ is the scale-invariant space for these coupled systems.

Our second motivation is to write the De Giorgi method in terms which make a direct connection with recent tools used in hyperbolic PDEs and make the universality of the formalism somehow remarkable. Namely, we have in mind the kinetic formulations for conservation laws [27] (see also [4, 5]), and level sets (the relation between level sets and kinetic formulations was already noticed in [14]).

The use of Stampachia truncations, which is fundamental in the De Giorgi method, was used for reaction-diffusion system for the first time in [1]. It was also used in [16, 7] to study the global regularity for some reaction-diffusion systems. The idea to replace the original method, which uses iterations on a discrete parameter, by the use of a continuous 'kinetic' parameter (and differentiation in this parameter) has already been used in the elliptic case in [28. Here we show it also applies to parabolic equations.

In order to motivate our method, we begin with the 'kinetic' proof of De Giorgi's result; Section 2 deals with the elliptic case and Section 3 with the parabolic case. With this material in hand, we can handle the case of system (1.1), which is done in Section 4

\section{De Giorgi method: elliptic case}

We illustrate our approach to the derivation of $L^{\infty}$ regularizing effects by the simpler case of elliptic equations. Let $u$ satisfy the inequality

$$
-\sum_{i, j=1}^{d} \frac{\partial}{\partial x_{i}}\left[a_{i j}(x) \frac{\partial}{\partial x_{j}} u\right] \leq f \in L^{p}\left(\mathbb{R}^{d}\right), \quad u_{+} \in L^{p^{\prime}}\left(\mathbb{R}^{d}\right), \quad \frac{1}{p}+\frac{1}{p^{\prime}}=1, \quad p>\frac{d}{2}
$$

in the 'kinetic' or 'entropy' sense (that is the related inequality holds for $(u-\xi)_{+}$), with $a_{i j}(x) \geq I d$ measurable. We wish to prove the standard result that $u$ is bounded above, namely

$$
u(x) \leq C_{p}\left(\|f\|_{p},\left\|(u)_{+}\right\|_{p^{\prime}}\right) .
$$

Step 1. For $\xi \geq 0$, we have (Sobolev injection for the first inequality and direct 
estimate on (2.1) for the second)

$$
\left\|(u-\xi)_{+}\right\|_{2 d /(d-2)}^{2} \leq C(d) \int_{\mathbb{R}^{d}}\left|\nabla(u-\xi)_{+}\right|^{2} \leq C(d)\|f\|_{p}\left\|(u-\xi)_{+}\right\|_{p^{\prime}} .
$$

Notice that because $p>\frac{d}{2}$, we have $p^{\prime}<\frac{d}{d-2}$ and $p^{\prime}<p^{\prime}+1<2 \frac{d-1}{d-2}<\frac{2 d}{d-2}$. Therefore, from (2.3) we conclude that $(u-\xi)_{+} \in L^{p^{\prime}+1}$ (and this leaves room for the case of dimension 2 using Gagliardo-Nirenberg-Sobolev inequality instead of the Sobolev inequality).

Step 2. Next, we claim that

$$
\frac{d}{d \xi} \int(u-\xi)_{+}^{p^{\prime}+1}=-\left(p^{\prime}+1\right) \int(u-\xi)_{+}^{p^{\prime}} \leq-C\left(\int(u-\xi)_{+}^{p^{\prime}+1}\right)^{\beta}
$$

with

$$
\beta=\frac{p^{\prime}}{p^{\prime}+1} \frac{2}{\theta+1}, \quad 0<\theta<1 .
$$

Indeed, this inequality follows from interpolation:

$$
\left\|(u-\xi)_{+}\right\|_{p^{\prime}+1} \leq\left\|(u-\xi)_{+}\right\|_{p^{\prime}}^{\theta}\left\|(u-\xi)_{+}\right\|_{2 d /(d-2)}^{(1-\theta)} \leq C(f)\left\|(u-\xi)_{+}\right\|_{p^{\prime}}^{\theta+(1-\theta) / 2},
$$

with

$$
\frac{1}{p^{\prime}+1}=\frac{\theta}{p^{\prime}}+(1-\theta) \frac{d-2}{2 d} .
$$

Step 3. It remains to notice that $\beta<1$ for $p>d / 2$. To prove it (i) notice that for $p=d / 2, p^{\prime}=d /(d-2)$, therefore $\frac{1}{p^{\prime}+1}=\frac{d-2}{d} \frac{1+\theta}{2}$ and $\beta=1$, (ii) for $p>d / 2$ then $\frac{d-2}{d}<\frac{1}{p^{\prime}}$ and thus $\frac{1}{p^{\prime}+1}<\frac{\theta}{p^{\prime}}+\frac{1-\theta}{2 p^{\prime}}=\frac{1}{p^{\prime}} \frac{1+\theta}{2}$.

Finally for $\xi=0$, the function $F(\xi):=\int(u-\xi)_{+}^{p^{\prime}+1}$ is bounded and the inequality

$$
F^{\prime}(\xi) \leq-C F(\xi)^{\beta}, \quad 0 \leq \beta<1,
$$

shows that $F(\cdot)$ vanishes for a finite value $\xi_{\max }$.

As mentioned earlier, 28, used this method and also proved Hölder regularity.

We can obtain the following explicit dependence on the norms of $f$ and $(u)_{+}$:

$$
\begin{aligned}
& u(x) \leq C_{p} \sqrt{\|f\|_{p}\left\|(u)_{+}\right\|_{p^{\prime}}}, \quad \text { for } d=2, \\
& u(x) \leq C_{p}\left(\left\|(u)_{+}\right\|_{p^{\prime}}^{2-d / p}\|f\|_{p}^{d / p^{\prime}}\right)^{\frac{1}{2-\frac{d}{p}+\frac{d}{p^{\prime}}}} \quad \text { for } d>2 .
\end{aligned}
$$

Indeed, consider for $\varepsilon, \lambda>0$,

$$
\begin{aligned}
u_{\varepsilon, \lambda}(x) & =\lambda u(\varepsilon x), \\
f_{\varepsilon, \lambda}(x) & =\varepsilon^{2} \lambda f(\varepsilon x) .
\end{aligned}
$$

This also solves (2.1) for the diffusion matrix $a_{i j}(\varepsilon x)$ which still satisfies $a_{i j}(\varepsilon x) \geq I d$. We choose $\varepsilon, \lambda$ such that

$$
\begin{aligned}
& \left\|\left(u_{\varepsilon, \lambda}\right)_{+}\right\|_{p^{\prime}}=1, \\
& \left\|f_{\varepsilon, \lambda}\right\|_{p}=1 .
\end{aligned}
$$


From above we have $u_{\varepsilon, \lambda}(x) \leq C_{p}$ for a universal constant $C_{p}$. So $u(x) \leq C_{p} / \lambda$ for any $x$. To compute $\lambda$, we check that (2.4) is equivalent to

$$
\begin{aligned}
\lambda \varepsilon^{-d / p^{\prime}}\left\|(u)_{+}\right\|_{p^{\prime}} & =1, \\
\lambda \varepsilon^{2-d / p}\|f\|_{p} & =1 .
\end{aligned}
$$

This leads to

$$
\begin{aligned}
& \lambda^{-1}=\sqrt{\|f\|_{p}\left\|(u)_{+}\right\|_{p^{\prime}}}, \quad \text { for } d=2, \\
& \lambda^{-1}=\left(\left\|(u)_{+}\right\|_{p^{\prime}}^{2-d / p}\|f\|_{p}^{d / p^{\prime}}\right)^{\frac{1}{2-\frac{d}{p}+\frac{d}{p^{\prime}}}} \quad \text { for } d>2 .
\end{aligned}
$$

\section{De Giorgi method: parabolic case}

Following the elliptic case we turn to the heat equation

$$
\left\{\begin{array}{l}
\frac{\partial u}{\partial t}-\sum_{i, j=1}^{d} \frac{\partial}{\partial x_{i}}\left[a_{i j}(x) \frac{\partial}{\partial x_{j}} u\right] \leq 0 \\
\left(u^{0}\right)_{+} \in L^{p}\left(\mathbb{R}^{d}\right)
\end{array}\right.
$$

For $\xi>0$, consider the 'energy'

$$
U(\xi)=\sup _{0 \leq t \leq \infty} \int_{\mathbb{R}^{d}}(u-\xi \eta(t))_{+}^{p} d x+4 \frac{p-1}{p} \int_{0}^{\infty} \int_{\mathbb{R}^{d}}\left|\nabla(u-\xi \eta(t))_{+}^{p / 2}\right|^{2} d x d t .
$$

The weight in time that will come out of our analysis is

$$
\eta(t)=t^{-\frac{d}{2 p}}, \quad 1 \leq p \leq \infty,
$$

and we will show that, for some $\xi_{1}>0, U\left(\xi_{1}\right)$ vanishes, which furnishes the regularizing effect in $L^{\infty}$ (strong hypercontractivity) via the estimate

$$
u(x, t) \leq \xi_{1} \eta(t) .
$$

Step 1. Because $\eta(0)=\infty$, elementary manipulations of Equation (3.1) give the energy estimate

$$
U(\xi) \leq 2 p \xi \int_{0}^{\infty} \int_{\mathbb{R}^{d}}|\dot{\eta}(t)|(u-\xi \eta(t))_{+}^{p-1} d x d t .
$$

Step 2. We prove the inequality

$$
\left(\int_{0}^{\infty} \int_{\mathbb{R}^{d}}(u-\xi \eta(t))_{+}^{q} d x d t\right)^{p / q} \leq C(d) U(\xi), \quad q=p \frac{d+2}{d},
$$

where $C(d)$ is a constant.

This follows from the Sobolev inequality with $r=2^{*} \frac{p}{2}, \frac{1}{2^{*}}=\frac{1}{2}-\frac{1}{d}$ (here again, in dimension 2 one should use Gagliardo-Nirenberg-Sobolev inequality)

$$
\int_{0}^{\infty}\left\|(u-\xi \eta(t))_{+}\right\|_{r}^{p} d t \leq C(d) \int_{0}^{\infty} \int_{\mathbb{R}^{d}}\left|\nabla(u-\xi \eta(t))_{+}^{p / 2}\right|^{2} d x d t
$$


or in other words

$$
\left\|(u-\xi \eta(t))_{+}\right\|_{L_{t}^{p}\left(L_{x}^{r}\right)} \leq C(d) U(\xi)^{1 / p},
$$

which we can interpolate with

$$
\left\|(u-\xi \eta(t))_{+}\right\|_{L_{t}^{\infty}\left(L_{x}^{p}\right)} \leq U(\xi)^{1 / p} .
$$

To get $L_{t}^{q} L_{x}^{q}$, we choose $q$ and $\bar{\theta}$ which satisfy

$$
\frac{1}{q}=\frac{\bar{\theta}}{p}+0, \quad \frac{1}{q}=\frac{\bar{\theta}}{r}+\frac{1-\bar{\theta}}{p},
$$

and thus

$$
\frac{1}{q}=\frac{p}{q} \frac{2}{p}\left(\frac{1}{2}-\frac{1}{d}\right)+\frac{q-p}{p q} \quad \Longrightarrow \quad \frac{q}{p}=2-2\left(\frac{1}{2}-\frac{1}{d}\right)=1+\frac{2}{d} .
$$

Step 3. We introduce a weight $\nu(t)$ that will be determined later on, and we define the function

$$
F(\xi)=\int_{0}^{\infty} \int_{\mathbb{R}^{d}} \nu(t)(u-\xi \eta(t))_{+}^{p} d x d t .
$$

We compute by interpolation, and use of steps 1 and 2,

$$
\begin{aligned}
F(\xi)^{1 / p} \leq & \left(\int_{0}^{\infty} \int_{\mathbb{R}^{d}} \nu(t)^{\frac{p-1}{p(1-\theta)}}(u-\xi \eta(t))_{+}^{p-1} d x d t\right)^{(1-\theta) /(p-1)} \\
& \times\left(\int_{0}^{\infty} \int_{\mathbb{R}^{d}}(u-\xi \eta(t))_{+}^{q} d x d t\right)^{\theta / q} \\
\leq & C \xi^{\theta / p}\left(\int_{0}^{\infty} \int_{\mathbb{R}^{d}}|\dot{\eta}(t)|(u-\xi \eta(t))_{+}^{p-1}\right)^{\frac{1-\theta}{p-1}+\frac{\theta}{p}} d x d t
\end{aligned}
$$

with $\frac{1}{p}=\frac{\theta}{q}+\frac{1-\theta}{p-1}$, which is also

$$
1=\theta \frac{2 p+d}{d+2}, \quad 0 \leq \theta \leq 1,
$$

and we need the compatibility conditions

$$
\nu(t)^{\frac{p-1}{p(1-\theta)}}=|\dot{\eta}(t)|=\nu(t) \eta(t)
$$

(the second equality will be used later on to obtain the correct $F^{\prime}$ ).

After a few computations (which are left to the reader) these two equalities define $\eta$ by the differential relation

$$
-\dot{\eta}(t)=\eta(t)^{\frac{p-1}{\theta p-1}}
$$

and $\frac{p-1}{\theta p-1}=1+\frac{2 p}{d}$. Its solution is indeed the negative power function (3.2), namely $\eta(t)=t^{-d /(2 p)}$. Notice that $\nu(t) \eta(t)=\eta(t)^{1+\frac{2 p}{d}}$, and thus

$$
\nu(t)=\eta(t)^{\frac{2 p}{d}}=\frac{1}{t} .
$$


Step 4. We have

$$
\begin{aligned}
\frac{d}{d \xi} F(\xi) & =-p \int_{0}^{\infty} \int_{\mathbb{R}^{d}} \nu(t) \eta(t)(u-\xi \eta(t))_{+}^{p-1} d x d t \\
& \leq-C \xi^{-\theta \beta} F(\xi)^{\beta}
\end{aligned}
$$

with

$$
\frac{1}{\beta}=\theta+(1-\theta) \frac{p}{p-1}=1+\frac{1-\theta}{p-1}>1, \quad 0<\beta<1,
$$

and because $\theta<1$,

$$
0<\theta \beta<1 .
$$

Therefore the function $F(\xi)$ vanishes in finite $\xi$.

Step 5. It remains to explain why $F$ is finite for some $\xi_{0}>0$ using $\nu(t)=1 / t$. When $u>2 \xi \eta$ and for $q>p$, we have $(u-\xi \eta(t))_{+}^{p-q}<(\xi \eta(t))^{p-q}$, hence the Tchebichev type inequality

$$
F(2 \xi) \leq \int_{0}^{\infty} \int_{\mathbb{R}^{d}} \frac{\nu(t)}{(\xi \eta(t))^{q-p}}(u-\xi \eta(t))_{+}^{q} d x d t<\infty .
$$

Since we have $q-p=\frac{2 p}{d}$, and using the exponents in (3.6), we arrive at

$$
\frac{\nu(t)}{(\xi \eta(t))^{q-p}}=1 / \xi^{q-p} .
$$

Therefore, for $\xi>0$, we have

$$
\begin{aligned}
F(2 \xi) & \leq \frac{1}{\xi^{q-p}} \int_{0}^{\infty} \int_{\mathbb{R}^{d}}(u-\xi \eta(t))_{+}^{q} d x d t \\
& \leq \frac{1}{\xi^{q-p}} \int_{0}^{\infty} \int_{\mathbb{R}^{d}} u_{+}^{q} d x d t \leq \frac{C}{\xi^{q-p}}\left\|\left(u^{0}\right)_{+}\right\|_{L^{p}\left(\mathbb{R}^{d}\right)}<\infty
\end{aligned}
$$

where the last inequality follows by the argument of step 2 choosing $\eta \equiv 1$ (then the energy is directly controlled by the initial data, in opposition to the case when $\eta(0)=\infty)$. The proof is complete.

Note that the estimates depend only on $\left\|\left(u^{0}\right)_{+}\right\|_{p}$. Hence, there exists a universal constant $C_{p}$ such that for $\bar{u}(x, t)=u(x, t) /\left\|\left(u^{0}\right)_{+}\right\|_{p}, \bar{u}(x, t) \leq C_{p} t^{\frac{-d}{2 p}}$. This allows us to sharpen (3.3) as

$$
u(x, t) \leq C_{p}\left\|\left(u^{0}\right)_{+}\right\|_{p} t^{\frac{-d}{2 p}} .
$$

\section{A nonlinear parabolic PDE arising in angiogenesis}

As mentioned in the introduction, the Keller-Segel system for chemotaxis has attracted a lot of studies mostly because solutions may blow-up for large mass. For small initial data global weak solutions exists (and many settings are possible) and gain of integrability is proved. For instance in [3] the authors prove, for initial mass below the critical mass, the gain of $L^{p}$ regularity for all $p \in(1, \infty)$ when the problem is set in $\mathbb{R}^{2}$ for the parabolic/elliptic problem. In [8, the parabolic/parabolic case in dimension larger than 3 is treated and $L^{p}$ integrability is reached with data just above the scale invariant exponents. This has been improved in [6] and in [21, 22]. The large time decay as $1 / t$ is also known in some cases; see [2] for the 2-d parabolic-elliptic Keller-Segel system with small mass. 
4.1. Setting the problem. The model reads

$$
\left\{\begin{array}{l}
\frac{\partial}{\partial t} n=\Delta n-\nabla \cdot[n \chi(c) \nabla c], \quad t>0, x \in \mathbb{R}^{d}, \\
\frac{\partial}{\partial t} c=-c n, \quad t>0, x \in \mathbb{R}^{d}, \\
n(0, x)=n_{0}(x) \geq 0, \quad c(0, x)=c_{0}(x) \geq 0, \quad x \in \mathbb{R}^{d} .
\end{array}\right.
$$

The sensitivity $\chi(c)$ is a given positive function on $\mathbb{R}_{+}$, generally chosen as a decreasing function since sensitivity is lower for higher concentrations of the chemical due to saturation effects.

Solutions to the angiogenesis system satisfy obvious a priori estimates for all $t \geq 0$ :

$$
\begin{gathered}
n(t) \geq 0, \quad 0 \leq c(t, x) \leq \max _{x \in \mathbb{R}^{d}} c_{0}(x), \\
\int_{\mathbb{R}^{d}} n(t, x)=M^{0}:=\int_{\mathbb{R}^{d}} n^{0}(x) .
\end{gathered}
$$

Moreover, when $\chi(c)$ is such that

$$
\mu:=\frac{1}{2} \inf _{c \geq 0}\left\{\frac{c \chi^{\prime}(c)}{\chi(c)}+1\right\}>0,
$$

system (4.1) satisfies an energy inequality given by

$$
\left\{\begin{array}{c}
\frac{d}{d t} \mathcal{E}(t) \leq-\int_{\mathbb{R}^{d}} n\left[|\nabla \ln (n)|^{2}+\mu|\nabla \Phi(c)|^{2}\right] \leq 0, \\
\mathcal{E}(t):=\int_{\Omega}\left[\frac{1}{2}|\nabla \Phi(c)|^{2}+n \ln (n)\right] \quad \text { and } \quad \Phi^{\prime}(c)=\sqrt{\frac{\chi}{c}} .
\end{array}\right.
$$

With these estimates the existence of weak solutions has been proved in [9].

Here, we are more interested in strong solutions in $L^{p}$ (see 24 for $H^{s}$ spaces). It is proved in [10] that there are strong solution in $L^{p}$, for appropriate $p \in[1, \infty)$ and for $\left\|n^{0}\right\|_{L^{\frac{d}{2}\left(\mathbb{R}^{d}\right)}}$ small enough, and in [11] that they are bounded in $L^{\infty}$ for an initial data in $L^{\infty}$. The regularizing effect, however, is open and this is what we want to prove here.

In the direction of strong solutions, another estimate can be proved:

$$
\|n(t)\|_{L^{\frac{d}{2}}\left(\mathbb{R}^{d}\right)} \leq\left\|n^{0}\right\|_{L^{\frac{d}{2}\left(\mathbb{R}^{d}\right)}} \quad \text { for } \quad\left\|n^{0}\right\|_{L^{\frac{d}{2}}\left(\mathbb{R}^{d}\right)} \leq K\left(d,\left\|c^{0}\right\|_{\infty}\right) \text { small enough. }
$$

This last estimate, borrowed from [10, requires an elementary computation that will be useful later, in a more general form, which we present now. It uses the following Nash-type inequality valid for $p>0, d \geq 2$ :

$$
\int_{\mathbb{R}^{d}} n^{p+1} \leq C(d, p)\left\|\nabla n^{p / 2}\right\|_{L^{2}\left(\mathbb{R}^{d}\right)}^{2}\|n\|_{L^{\frac{d}{2}}\left(\mathbb{R}^{d}\right)} .
$$

For $d>2$, the proof uses the Sobolev inequality:

$$
\int_{\mathbb{R}^{d}} n^{p+1} \leq\left\|n^{p / 2}\right\|_{L^{\frac{2 d}{d-2}}\left(\mathbb{R}^{d}\right)}^{2}\|n\|_{L^{\frac{d}{2}}\left(\mathbb{R}^{d}\right)} \leq C(d)\left\|\nabla n^{p / 2}\right\|_{L^{2}\left(\mathbb{R}^{d}\right)}^{2}\|n\|_{L^{\frac{d}{2}}\left(\mathbb{R}^{d}\right)}
$$


For $d=2$, we get the result in the following way:

$$
\begin{aligned}
\int_{\mathbb{R}^{2}} n^{p+1} \leq\left\|\nabla n^{\frac{p+1}{2}}\right\|_{L^{1}\left(\mathbb{R}^{2}\right)}^{2} & \leq C(p)\left\|n^{1 / 2} \nabla n^{p / 2}\right\|_{L^{1}\left(\mathbb{R}^{2}\right)}^{2} \\
& \leq C(p)\left\|\nabla n^{p / 2}\right\|_{L^{2}\left(\mathbb{R}^{2}\right)}^{2}\|n\|_{L^{1}\left(\mathbb{R}^{2}\right)} .
\end{aligned}
$$

We define $\phi$ by

$$
\phi^{\prime}(c)=\phi(c) \chi(c), \quad c>0, \quad \phi(0)=1,
$$

and compute, following [10], for any dimension $d \geq 2$,

$$
\begin{aligned}
\frac{d}{d t} \int_{\mathbb{R}^{d}}\left(\frac{n}{\phi(c)}-K\right)_{+}^{p} \phi(c)=- & 4 \frac{p-1}{p} \int_{\mathbb{R}^{d}} \phi(c)\left|\nabla\left(\frac{n}{\phi(c)}-K\right)_{+}^{p / 2}\right|^{2} \\
& +(p-1) \int_{\mathbb{R}^{d}} \phi^{2}(c) \chi(c) c\left(\frac{n}{\phi(c)}-K\right)_{+}^{p+1} \\
& +(2 p-1) K \int_{\mathbb{R}^{d}} \phi^{2}(c) \chi(c) c\left(\frac{n}{\phi(c)}-K\right)_{+}^{p} \\
& +p K^{2} \int_{\mathbb{R}^{d}} \phi^{2}(c) \chi(c) c\left(\frac{n}{\phi(c)}-K\right)_{+}^{p-1}
\end{aligned}
$$

Therefore, we also have

$$
\begin{aligned}
& \frac{d}{d t} \int_{\mathbb{R}^{d}}\left(\frac{n}{\phi(c)}-K\right)_{+}^{p} \phi(c) \\
= & -4 \frac{p-1}{p}\left[\int_{\mathbb{R}^{d}} \phi(c)\left|\nabla\left(\frac{n}{\phi(c)}-K\right)_{+}^{p / 2}\right|^{2}-\frac{p}{4^{2}} \phi^{2}(c) \chi(c) c\left(\frac{n}{\phi(c)}-K\right)_{+}^{p+1}\right] \\
& +(2 p-1) K \int_{\mathbb{R}^{d}} \phi^{2}(c) \chi(c) c\left(\frac{n}{\phi(c)}-K\right)_{+}^{p}+p K^{2} \int_{\mathbb{R}^{d}} \phi^{2}(c) \chi(c) c\left(\frac{n}{\phi(c)}-K\right)_{+}^{p-1} .
\end{aligned}
$$

From this equality, we deduce two useful inequalities.

On the one hand, with $K=0$, the Nash inequality (4.7) gives

$$
\begin{aligned}
\frac{d}{d t} \int_{\mathbb{R}^{d}}\left(\frac{n}{\phi(c)}\right)^{p} \phi(c) & =-4 \frac{p-1}{p}\left[\int_{\mathbb{R}^{d}} \phi(c)\left|\nabla\left(\frac{n}{\phi(c)}\right)^{p / 2}\right|^{2}-\frac{p}{4^{2}} \phi^{2}(c) \chi(c) c\left(\frac{n}{\phi(c)}\right)^{p+1}\right] \\
& \leq 4 \frac{p-1}{p} \int_{\mathbb{R}^{d}}\left|\nabla\left(\frac{n}{\phi(c)}\right)^{p / 2}\right|^{2}\left[1-C\left(d, p,\|c\|_{\infty}\right)\left\|\frac{n}{\phi(c)}\right\|_{d / 2}\right]
\end{aligned}
$$

which, with $p=d / 2$, explains the a priori estimate (4.6). Also we conclude (with a stronger smallness assumption if needed)

$$
2 \frac{p-1}{p} \int_{0}^{T} \int_{\mathbb{R}^{d}} \phi(c)\left|\nabla\left(\frac{n}{\phi(c)}\right)^{p / 2}\right|^{2}+\int_{\mathbb{R}^{d}}\left(\frac{n(T)}{\phi(c(T))}\right)^{p} \phi(c(T)) \leq \int_{\mathbb{R}^{d}}\left(\frac{n^{0}}{\phi\left(c^{0}\right)}\right)^{p} \phi\left(c^{0}\right) .
$$

On the other hand, still under this smallness condition in (4.6), we have, for any 
$\max \left\{1, \frac{d}{2}-1\right\} \leq p<\infty$,

$$
\begin{aligned}
\frac{d}{d t} \int_{\mathbb{R}^{d}}\left(\frac{n}{\phi(c)}-K\right)_{+}^{p} \phi(c) \leq- & 2 \frac{p-1}{p} \int_{\mathbb{R}^{d}} \phi(c)\left|\nabla\left(\frac{n}{\phi(c)}-K\right)_{+}^{p / 2}\right|^{2} \\
& +(2 p-1) K \int_{\mathbb{R}^{d}} \phi^{2}(c) \chi(c) c\left(\frac{n}{\phi(c)}-K\right)_{+}^{p} \\
& +p K^{2} \int_{\mathbb{R}^{d}} \phi^{2}(c) \chi(c) c\left(\frac{n}{\phi(c)}-K\right)_{+}^{p-1} .
\end{aligned}
$$

4.2. Regularizing effects in $L^{\infty}$. It is our purpose to prove here our main result.

TheOREM 4.1. For $c^{0} \in L^{\infty}\left(\mathbb{R}^{d}\right)$ and $\left\|n^{0}\right\|_{L^{d / 2}\left(\mathbb{R}^{d}\right)} \leq C\left(d,\left\|c^{0}\right\|_{\infty}\right)$ small enough, the smooth solutions to 4.1) satisfy, for any $T>0$,

1. If $n^{0} \in L^{\infty}$ then $n \in L^{\infty}\left((0, T) \times \mathbb{R}^{d}\right)$,

2. if $n^{0} \in L^{p}\left(\mathbb{R}^{d}\right)$ with $p>\frac{d+2}{2}$, then $\|n(t)\|_{L^{\infty}\left(\mathbb{R}^{d}\right)} \leq C(T) t^{-\frac{d}{2 p}}, 0<t \leq T$, (the rate of the heat equation),

3. if $n^{0} \in L^{p}\left(\mathbb{R}^{d}\right)$ with $p>\frac{d(d+4)}{2(d+2)}$, then $\|n(t)\|_{L^{\infty}\left(\mathbb{R}^{d}\right)} \leq \frac{C(T)}{t}, 0<t \leq T$, (a rate weaker than for the heat equation),

4. if $n^{0} \in L^{d / 2}\left(\mathbb{R}^{d}\right)$, then $\|n(t)\|_{L^{\infty}\left(\mathbb{R}^{d}\right)} \leq \frac{C\left(d,\left\|c^{0}\right\|_{\infty}\right)}{t}$ for $t>0$.

In particular, this theorem implies some kind of remarkable regularizing effect on $c$, even though it is driven by an ODE, because such integrability of $n$ in $L^{\infty}$ is not true for all bounded drifts $c(t, x)$.

Also, the quadratic term in the model does not seem to have an effect on the regularizing effects as this is the case for the long time decay [29, 8 .

The first result is from [11] and we will not prove it again.

Proof. (Second estimate.) We follow the case of the heat equation, and in the different steps we consider the additional terms coming from the energy inequality. One of the consequences is that we have to work on a finite time interval $(0, T)$.

Step 1. We define, with $C=2 \frac{p-1}{p} \frac{\phi_{\max }}{\phi_{\min }}$,

$$
U(\xi)=\sup _{0 \leq t \leq T} \int_{\mathbb{R}^{d}}\left(\frac{n}{\phi(c)}-\xi \eta(t)\right)_{+}^{p}+C \int_{0}^{T} \int_{\mathbb{R}^{d}}\left|\nabla\left(\frac{n}{\phi(c)}-\xi \eta(t)\right)_{+}^{p / 2}\right|^{2}
$$

and we first deduce after integrating (4.11) that, still under the condition $\eta(0)>$ 


$$
\begin{aligned}
& \left\|n^{0}\right\|_{\infty}, \\
& \phi_{\min } U(\xi) \leq \sup _{0 \leq t \leq T} \int_{\mathbb{R}^{d}}\left(\frac{n}{\phi(c)}-\xi \eta(t)\right)_{+}^{p} \phi(c)+2 \frac{p-1}{p} \int_{0}^{T} \int_{\mathbb{R}^{d}} \phi(c)\left|\nabla\left(\frac{n}{\phi(c)}-\xi \eta(t)\right)_{+}^{p / 2}\right|^{2} \\
& \leq-\xi \int_{0}^{T} \dot{\eta}(t) \int_{\mathbb{R}^{d}}\left(\frac{n}{\phi(c)}-\xi \eta(t)\right)_{+}^{p-1} \phi(c) \\
& +(2 p-1) \int_{0}^{T} \xi \eta(t) \int_{\mathbb{R}^{d}} \phi^{2}(c) \chi(c) c\left(\frac{n}{\phi(c)}-\xi \eta(t)\right)_{+}^{p} \\
& \quad+p \int_{0}^{T}(\xi \eta(t))^{2} \int_{\mathbb{R}^{d}} \phi^{2}(c) \chi(c) c\left(\frac{n}{\phi(c)}-\xi \eta(t)\right)_{+}^{p-1}
\end{aligned}
$$

Step 2. On the other hand the Sobolev inequality still gives, as in (3.4) for the linear case,

$$
\left(\int_{0}^{T} \int_{\mathbb{R}^{d}}\left(\frac{n}{\phi(c)}-\xi \eta(t)\right)_{+}^{q} d x d t\right)^{p / q} \leq C U(\xi), \quad q=p \frac{d+2}{d} .
$$

Step 3. We again introduce a weight $\nu(t)$ to be determined later on, and define

$$
F(\xi)=\int_{0}^{T} \int_{\mathbb{R}^{d}} \nu(t)\left(\frac{n}{\phi(c)}-\xi \eta(t)\right)_{+}^{p} d x d t
$$

We get

$$
\begin{gathered}
F(\xi)^{1 / p} \leq\left(\int_{0}^{T} \int_{\mathbb{R}^{d}} \nu(t)^{\frac{p-1}{p(1-\theta)}}\left(\frac{n}{\phi(c)}-\xi \eta(t)\right)_{+}^{p-1} d x d t\right)^{(1-\theta) /(p-1)} \\
\times\left(\int_{0}^{T} \int_{\mathbb{R}^{d}}\left(\frac{n}{\phi(c)}-\xi \eta(t)\right)_{+}^{q} d x d t\right)^{\theta / q}
\end{gathered}
$$

or equivalently, using (4.13),

$$
F(\xi)^{1 / p} \leq[U(\xi)]^{\theta / p}\left(\int_{0}^{T} \int_{\mathbb{R}^{d}} \nu(t)^{\frac{p-1}{p(1-\theta)}}\left(\frac{n}{\phi(c)}-\xi \eta(t)\right)_{+}^{p-1} d x d t\right)^{(1-\theta) /(p-1)}
$$

still with

$$
\theta=\frac{d+2}{d+2 p}
$$

At this stage, we impose that there is a constant $C(T)$ (for this it might be necessary to work with $T$ finite):

$$
\left\{\begin{array}{l}
\nu(t)^{\frac{p-1}{p(1-\theta)}}+|\dot{\eta}(t)| \leq C(T) \nu(t) \eta(t) \\
\eta(t) \leq C(T) \nu(t), \quad \dot{\eta}(t) \leq 0
\end{array}\right.
$$

We can take, for instance (although another choice is made later),

$$
\nu(t)=\frac{1}{t}, \quad \eta(t)=t^{-\frac{d}{2 p}}, \quad p \geq d / 2,
$$


with $C(T)=T^{1-\frac{d}{2 p}}$. In the case at hand, because $\frac{p-1}{p(1-\theta)}=1+\frac{d}{2 p}$, the constraint $T<\infty$ only comes from the necessity to fulfill the second line in (4.15).

We can then write (4.12) as

$$
U(\xi) \leq C \xi\left(-F^{\prime}(\xi)\right)+C \xi F(\xi)+C \xi^{2}\left(-F^{\prime}(\xi)\right),
$$

and we can write (4.14) as

$$
F(\xi) \leq[U(\xi)]^{\theta}\left(-F^{\prime}(\xi)\right)^{(1-\theta) p /(p-1)} .
$$

Step 4. Then we combine (4.17) and (4.18) to obtain with

$$
\begin{gathered}
p^{\prime}=\frac{p}{p-1}, \\
F(\xi)^{1 / \theta} \leq C\left(-F^{\prime}(\xi)\right)^{\frac{(1-\theta) p^{\prime}}{\theta}}\left[\xi\left(-F^{\prime}(\xi)\right)+\xi F(\xi)+\xi^{2}\left(-F^{\prime}(\xi)\right)\right],
\end{gathered}
$$

and because we only consider this differential inequality for $\xi \geq \xi_{0}>0$,

$$
F(\xi)^{1 / \theta} \leq C\left(-F^{\prime}(\xi)\right)^{\frac{(1-\theta) p^{\prime}}{\theta}}\left[\xi F(\xi)+\xi^{2}\left(-F^{\prime}(\xi)\right)\right] .
$$

Furthermore, still with

$$
\frac{1}{\beta}=1+\frac{1-\theta}{p-1}>1, \quad 0<\beta<1,
$$

we have the differential inequality

$$
1 \leq C\left(\frac{-F^{\prime}(\xi)}{F(\xi)^{\beta}}\right)^{\frac{(1-\theta) p^{\prime}}{\theta}}\left[\xi F(\xi)^{1-\beta}+\xi^{2} \frac{-F^{\prime}(\xi)}{F(\xi)^{\beta}}\right] .
$$

We may use $G(\xi)=F(\xi)^{1-\beta}$ instead, the result of which would read

$$
\begin{gathered}
1 \leq C\left(-G^{\prime}(\xi)\right)^{\frac{(1-\theta) p^{\prime}}{\theta}}\left[\xi G(\xi)+\xi^{2}\left(-G^{\prime}(\xi)\right)\right], \\
\xi^{2}\left(-G^{\prime}(\xi)\right)^{\frac{1}{\beta \theta}}+\xi G(\xi)\left(-G^{\prime}(\xi)\right)^{\frac{1}{\beta \theta}-1} \geq c, \\
\xi^{2 \beta \theta}\left(-G^{\prime}(\xi)\right)+\xi^{\beta \theta} G(\xi)^{\beta \theta}\left(-G^{\prime}(\xi)\right)^{1-\beta \theta} \geq c>0 .
\end{gathered}
$$

which is equivalent to

$$
G^{\prime}(\xi) \leq-c \min \left(\xi^{-2 \beta \theta},[\xi G(\xi)]^{\frac{\beta \theta}{1-\beta \theta}}\right) .
$$

We recall that we always have $0<\beta \theta<1$. The term in $\xi G(\xi)$ is bad in the right hand side of (4.19) and we have to assume that $p$ is such that

$$
2 \beta \theta<1 \quad \Longleftrightarrow \quad \frac{1}{\theta \beta}=1+\frac{2 p}{d+2}>2 .
$$


This condition also gives the possible exponents in our proof:

$$
p>\frac{d+2}{2} .
$$

Then we may built supersolutions of the ODE (4.19):

$$
\bar{G}(\xi)=A-B \xi^{1-2 \beta \theta}, \quad B>0 \text { small enough. }
$$

We choose $A$ large enough so as to impose $G\left(\xi_{0}\right)<A-B \xi_{0}^{1-2 \beta \theta}$ (see step 5). Therefore $G(\xi) \leq A-B \xi^{1-2 \beta \theta}$ and thus $G$ (or equivalently $F$ ) vanishes for a finite $\xi_{1}$.

Step 5. We have $G\left(\xi_{0}\right)<\infty$ for some $\xi_{0}$ by the Tchebichev argument of Section 3 , which holds true here because we have handled the same weights $\eta$ and $\nu$. The proof of the second inequality is completed.

Proof. (Third estimate.) We use the same proof. But, in order to extend the range of validity for initial integrability, we choose in (4.15) the weights

$$
\eta(t)=\nu(t)=1 / t,
$$

and the constant $C(T)$ comes now from the first inequality in (4.15). We choose also $p>(d+2) / 2$ according to (4.20) so that the above proof holds true. But we modify Step 5 as follows.

(Step 5-modified.) By a Tchebichev type inequality we have

$$
F(2 \xi) \leq \int_{0}^{\infty} \int_{\mathbb{R}^{d}} \frac{\nu(t)}{(\xi \eta(t))^{\bar{q}-p}}(n-\xi \eta(t))_{+}^{\bar{q}} d x d t<\infty .
$$

We choose $\bar{q}=p+1$ so that the exponents in $t$ cancel and we arrive at

$$
F(2 \xi) \leq \frac{1}{\xi} \int_{0}^{\infty} \int_{\mathbb{R}^{d}}(n-\xi \eta(t))_{+}^{\bar{q}} d x d t \leq \frac{1}{\xi} \int_{0}^{\infty} \int_{\mathbb{R}^{d}} n^{\bar{q}} d x d t \leq \frac{C}{\xi}\left\|n^{0}\right\|_{L^{\bar{p}}\left(\mathbb{R}^{d}\right)}<\infty,
$$

with

$$
\bar{p}=\bar{q} \frac{d}{d+2}=(p+1) \frac{d}{d+2}<\frac{d+4}{2} \frac{d}{d+2},
$$

by the Sobolev injection argument of step 2 of Section 3 and by using the a priori bound (4.10) (with $\bar{p}$ in place of $p$ ).

The proof is complete.

Proof. (Fourth estimate.) We use the fact that the norms $\left\|n^{0}\right\|_{L^{d / 2}}$ and $\left\|c^{0}\right\|_{L^{\infty}}$ are scale invariant. Let us first show the estimate for $T=2$. Let $\left\|n^{0}\right\|_{L^{d / 2}}$ be small enough. Then from the a priori bound (4.10) with $p=d / 2$, and still with the Sobolev inequality as in (3.4) for the linear case, we have

$$
\int_{0}^{1} \int_{\mathbb{R}^{d}} n^{\frac{d+2}{2}} d x d t \leq C
$$

Therefore there is a $t_{0} \leq 1$ for which $\int n\left(t_{0}\right)^{\frac{d+2}{2}} d x \leq C$, and the decay property in (4.10) gives

$$
\int_{\mathbb{R}^{d}} n(1)^{\frac{d+2}{2}} d x \leq C .
$$


Since

$$
\frac{d+2}{2}>\frac{d}{2} \frac{d+4}{d+2}
$$

then from the third decay result, departing from 1 we conclude that for $1<t$

$$
\|n(t)\|_{L^{\infty}\left(\mathbb{R}^{d}\right)} \leq C\left(\int_{\mathbb{R}^{d}} n(1)^{\frac{d+2}{2}} d x\right) \frac{1}{t-1} .
$$

In particular, for $t=2$,

$$
\|n(2)\|_{L^{\infty}\left(\mathbb{R}^{d}\right)} \leq \tilde{C} .
$$

Note that the constant $\tilde{C}$ depends only on $\left\|n^{0}\right\|_{L^{d / 2}}$ and $\left\|c^{0}\right\|_{L^{\infty}}$.

Consider now

$$
n_{R}(t, x)=R^{2} n\left(R^{2} t, R x\right), \quad c_{R}(t, x)=c\left(R^{2} t, R x\right) .
$$

The scales have been chosen because $\left(n_{R}, c_{R}\right)$ satisfies the same equation (1.1) with initial value

$$
n_{R}(0, x)=R^{2} n^{0}(R x), \quad c_{R}(0, x)=c^{0}(R x) .
$$

The critical exponents are such that

$$
\left\|n_{R}^{0}\right\|_{L^{d / 2}}=\left\|n^{0}\right\|_{L^{d / 2}}, \quad\left\|c_{R}^{0}\right\|_{L^{\infty}}=\left\|c^{0}\right\|_{L^{\infty}} .
$$

We can then obtain at $t=2$ the same inequality with the same constant $\tilde{C}$ :

$$
\left\|n_{R}(2)\right\|_{L^{d / 2}} \leq \tilde{C}
$$

which leads to

$$
\left\|n\left(2 R^{2}\right)\right\|_{L^{\infty}} \leq \frac{2 \tilde{C}}{2 R^{2}} .
$$

Since the estimate is valid for any $R>0$, the fourth result follows.

\section{REFERENCES}

[1] N.D. Alikakos, $L^{p}$ bounds of solutions of reaction-diffusion equations, Commun. PDE, 4(8), 827-868, 1979.

[2] A. Blanchet, J. Dolbeault, M. Escobedo, and J. Fernandez, Asymptotic behaviour for small mass in the two-dimensional parabolic-elliptic Keller-Segel model, J. Math. Anal. Appl., 361, 533-542, 2010.

[3] A. Blanchet, J. Dolbeault, and B. Perthame, Two-dimensional Keller-Segel model: Optimal critical mass and qualitative properties of the solutions, Elec. J. Diff. Equ., 1-32, 2006.

[4] Y. Brenier, Averaged multivalued solutions for scalar conservation laws, SIAM J. Numer. Anal., 21, 1013-1037, 1984.

[5] Y. Brenier, Résolution d'équations d'évolution quasilinéaires en dimension $N$ d'espace à l'aide d'équations linéaires en dimension N+1, J. Differ. Equs., 50, 375-390, 1983.

[6] V. Calvez and L. Corrias, The parabolic/parabolic Keller-Segel model in $\mathbb{R}^{2}$, Commun. Math. Sci., 6(2), 417-447, 2008.

[7] C. Caputo and A. Vasseur, Global regularity of solutions to systems of reaction-diffusion with Sub-Quadratic Growth in any dimension, Commun. PDE, 34, 10-12, 1228-1250, 2009. 
[8] L. Corrias, and B. Perthame, Asymptotic decay for the solutions of the parabolic-parabolic Keller-Segel chemotaxis system in critical spaces, Mathematical and Computer Modelling, 47(7-8), 755-764, 2008.

[9] L. Corrias, B. Perthame, and H. Zaag, A chemotaxis model motivated by angiogenesis, C.R. Acad. Sc. Paris, Série I., 336/2, 141-146, 2003.

[10] L. Corrias, B. Perthame, and H. Zaag, Global Solutions of some chemotaxis and angiogenesis systems in high space dimensions, Milano J. Math., 72, 1-29, 2004.

[11] L. Corrias, B. Perthame, and H. Zaag, $L^{p}$ and $L^{\infty}$ a priori estimates for some chemotaxis models and applications to the Cauchy problem, The Mechanism of the Spatio-Temporal Pattern Arising in Reaction Diffusion System, Kyoto, 2004.

[12] F.A. Davidson, A.R.A. Anderson, and M.A.J. Chaplain, Steady-state solutions of a generic model for the formation of capillary networks, Appl. Math. Lett., 13, 127-132, 2000.

[13] E. De Giorgi, Sulla differenziabilità e l'analiticità delle estremali degli integrali multipli regolari, Mem. Accad. Sci. Torino. Cl. Sci. Fis. Mat. Nat., (3), 25-43, 1957.

[14] L.C. Evans, A survey of entropy methods for partial differential equations, Bulletin AMS, 41, 409-438, 2004.

[15] M.A. Fontelos, A. Friedman, and B. Hu, Mathematical analysis of a model for the initiation of angiogenesis, SIAM J. Math. Anal., 33, 1330-1355, 2002.

[16] T. Goudon and A. Vasseur, Regularity analysis for systems of reaction-diffusion equations, Ann. Sci. Ec. Norm. Super., 43(4), 1, 117-14, 2010.

[17] W. Jäger and S. Luckhaus, On explosions of solutions to a system of partial differential equations modelling chemotaxis, Trans. Amer. Math. Soc., 329, 819-824, 1992.

[18] K. Kang and Juan J.L. Velazquez, Qualitative behavior of a Keller-Segel model with nondiffusive memory, Commun PDE, 35(2), 245-274, 2010.

[19] E.F. Keller and L.A. Segel, Model for chemotaxis, J. Theor. Biol., 30, 225-234, 1971.

[20] H. Kozono and Y. Sugiyama, Local existence and finite time blow-up in the 2-D KellerSegel system, J. Evol. Equ., 8, 353-378, 2008.

[21] H. Kozono and Y. Sugiyama, Global strong solution to the semi-linear Keller-Segel system of parabolic-parabolic type with small data in scale invariant spaces, J. Differ. Equs., 247, 1-32, 2009.

[22] H. Kozono and Y. Sugiyama, Strong solutions to the Keller-Segel system with the weak $L^{\frac{n}{2}}$ initial data and its application to the blow-up rate, Math. Nachr., 283, 732-751, 2010.

[23] H.A. Levine, M. Nilsen-Hamilton, and B.D. Sleeman, Mathematical modelling of the onset of capillary formation initiating angiogenesis, J. Math. Biol., 42, 195-238, 2001.

[24] D. Li, T. Li, and K. Zhao, On a hyperbolic-parabolic system modeling repulsive chemotaxis, Math. Mode. Meth. Appl. Sci., 21(8), to appear, August 2011.

[25] T. Nagai, Blow-up of radially symmetric solutions to a chemotaxis system, Adv. Math. Sci. Appl., 5, 581-601, 1995.

[26] T. Nagai, Blowup of nonradial solutions to parabolicelliptic systems modeling chemotaxis in two-dimensional domains, J. Inequal. Appl., 6, 37-45, 2001.

[27] B. Perthame, Kinetic Formulation of Conservation Laws, Oxford Lecture Series in Mathematics and its Applications, Oxford University Press No., 21, 2002.

[28] P. Tilli, Remarks on the Hölder continuity of solutions to elliptic equations in divergence form, Calc. Var., 25(3), 395-401, 2006.

[29] M. Winkler, Aggregation vs global diffusive behavior in the higher-dimensional Keller-Segel model, J. Differ. Equs., 248(12), 2889-2905, 2010. 